Article

\title{
Emergence as Mesoscopic Coherence
}

\section{Gianfranco Minati $^{1} *$ and Ignazio Licata ${ }^{2}$}

1 Italian Systems Society, Via Pellegrino Rossi 42 Milan, I-20161, Italy

2 School of Advanced International Studies on Theoretical and non Linear Methodologies of Physics, Bari I-70124, Italy; E-Mail: Ignazio.licata@ejtp.info

* Author to whom correspondence should be addressed; E-Mail: gianfranco.minati@ AIRS.it.

Received: 2 August 2013; in revised form: 15 September 2013 / Accepted: 18 September 2013 / Published: 27 September 2013

\begin{abstract}
We propose here a formal approach to study collective behaviors intended as coherent sequences of spatial configurations, adopted by agents through various corresponding structures over time. Multiple, simultaneous structures over time and their sequences are called Meta-Structures and establish sequences of spatial configurations considered as emergent on the basis of coherent criteria chosen and detected by an observer. This coherence is represented by patterns of values of the proper mesoscopic variables adopted, i.e., meta-structural properties. We introduce a formal tool, i.e., the family of mesoscopic general vectors, defined by the observer, able to detect coherent behaviors like ergodic or quasi-ergodic ones. Such approach aims to provide a general framework to study intrinsically stochastic processes where the "universal evolution laws" fail. However, at the same, the system is structured enough to show significant clusters of collective behaviors "invisible to" simple statistics.
\end{abstract}

Keywords: clustering; collective beings; emergence; ideal and non-ideal models for complex systems; mesoscopic level of system description; quasi-ergodic behavior

\section{Introduction}

Understanding what "mechanism" allows a system to maintain its coherence and its global robustness is fundamental to study collective behaviors. The general approach usually adopt models already well consolidated in physics, assuming strong hypotheses on the dynamics of the system. For the most part, such models are inspired by classical and quantum theories of phase transitions and 
are thus termed as ideal models [1-4]. However, these approaches are still unsatisfactory for many research fields, such as socio-economic agent-based systems, and some biological and cognitive phenomena. Alternative approaches are based on another class of models termed as non-ideal, for instance, cellular automata, genetic algorithms and neural networks. This class of models focuses on fitness, power laws, scale-freeness, and topology [5-10].

Ideal models are based on general principles and studied analytically in an infinite volume independent on initial conditions and boundaries. Non-ideal models are based on opposite assumptions, considering lucky choices and studied through computer simulations. It is possible to say that ideal models follow a top-down approach while non-ideal models follow bottom-up approaches. The possibility of integrating the two approaches is currently under discussion. We propose here an approach based on the philosophy of the "middle way" [11]. Let us start from two main considerations:

(a) Within the mesoscopic description level of complex processes, areas of continuous negotiations between micro and macro, it is impossible in principle-because the problem becomes intractable - to consider all the dynamic inter-relations between agents and between system and environment. We have to keep in mind that the concept of "mesoscopic variable" can mean many things. In the systems where it is possible to apply the ideal models - for ex Haken Synergetics [12] - identifying the "right" mesoscopic variables is suggested by the same system's dynamics. In other cases, such as very fast dynamics between agents where the role of the hubs change rapidly, the identifying of mesoscopic variables is not so patent. Also for non-ideal models it is necessary to do some a priori assumptions on the system's dynamics, for example: choosing rules for cellular automata and weights for neural nets. In many cases of natural or artificial populations it is impossible. However, they are phenomena showing their peculiar regularity. Therefore, we have changed the strategy to approach them by reducing, at minimum, the a priori hypotheses, to observe the system over time and so to acquire a wide sequence of historical data. On the basis of these data it will be possible to make a posteriori conjectures on the more suitable mesoscopic variables to describe the significant patterns of the system.

(b) In these irreducible cases it is on the observer to define the mesoscopic variables, which could be the clue of dynamics, even if not so "clean" as in ideal models. It is necessary to underline that this approach is different from statistics - which gives an important contribution for the study of the single variables and their correlations-because the strategy is purely constructivist: it is the observer's choice to decide which global configurations bring significant information and which have to be considered just as noise, on the basis of its own operative choices on system and environment. In another paper we called such strategy as "without Physics" [13] just for the switching of the a priori and a posteriori and for the accent on the observer's role. We have to select from several families of possible observables and simultaneously, consider mesoscopically some of them as being clustered by the particular aspects the researcher defined when adopting a research strategy. We expect the identification of structural changes, similar in a way to phase transitions. As a matter of fact, the characteristic of collective systems, when adopting collective behaviors, is precisely their ability to rearrange through dynamic sequences of local emergent relationships and ways of 
interacting, based on the emergence of local and temporary constraints, which are an integral part of the system's dynamics that make it unpredictable. The idea is to use and superimpose several mathematical grids, i.e., precise meta-structures, in order to detect emergence as mesoscopic coherence, for instance, by statistical regularities such as possible ergodicity or quasi-ergodicity [14,15].

By the classification of forms of emergence introduced by Crutchfield [16], we note that meta-structures may be considered as an attempt of synthesis between ideal and non-ideal approaches. In fact, it is an "opportunistic", bottom up and a posteriori approach, which does not renounce to search for models of general collective behaviors.

Actually, the selection of observables is performed here by the observer and is intended as collective design [17]. In this way, acting through collective variables tends to detect emergence as novelty [18-20].

Here, to reaffirm these concepts, we consider collective behaviors modeled by variations of geometrical coordinates and spatial properties, e.g., speed, direction, distance and topology of interacting generic agents of Collective Systems represented. Also, by variations in shapes, density, volume and behavior of the collective entity, e.g., swarms, flocks, and protein chains emerging in 3D space. We refer to such collective behaviors as Spatial Collective Behaviors (SCBs) adopted by Collective Systems. The extension of the approach to non-spatial collective behaviors requires adoption of different mesoscopic variables, whereas meta-structural properties should, in any case, represent the coherence deriving from processes of emergence.

The novel ideas in our approach consist of considering SCBs as being established through coherent sequences of spatial configurations, adopted by interacting agents through various corresponding structures over time. The corresponding sequences of new structures are intended here as sequences of phase transitions and their coherence is modeled by using properties of values taken by suitable mesoscopic variables, rather than micro or macroscopic ones as in more usual approaches, where the term configuration denotes the spatial arrangement of agents. We introduce approaches considering emergence as mesoscopic coherence.

\section{Collective Systems}

It is well known that systems may be studied by using a variety of assumptions. Our research focuses upon so-called collective systems, established by permanently interacting generic agents provided or not provided with cognitive systems [21,22]. By generic agent, we consider any entity able to interact with another, i.e., influencing the other's behavior, for instance, by exchange of energy/information, e.g., through collisions, goods in economy, and chemical or behavioral signals. We underline that this is considered as a classical approach, since the assumptions of locality and separability in classical time are always valid.

Consider the following cases:

(a) Examples of collective systems, given by the collective motion of living systems, provided with sufficiently complex cognitive systems, include flocking, swarming, anthills, herding, schooling, crowd, and traffic. In these cases, the interactions involve cognitive processing. 
(b) Examples of collective systems, given by the collective motion of living systems, provided with no cognitive systems, include amoeba, bacterial colonies, cells, and macromolecules. Examples of collective systems, given by the collective motion of non-living systems, include lasers, systems of boats, nano-swimmers, nematic fluids, networks, signaling traffic, rods on vibrating surfaces, shaken metallic rods - interaction involves reacting — and simple robots [10]. Interaction is given by simple artificial cognitive systems.

(c) Examples of collective artificial systems, given by collective interactions of various natures other than physical motion include, communities of mobile phone networks, industrial districts, markets, morphological properties of cities and urban development, networks like the Internet, and queues. Interaction is given by systems of cognitive processing and reactions.

Several cases and distinctions should be considered:

- The homogeneous and non-homogeneous cases where generic agents have or do not have the same properties used for interacting. In the first case, we will consider populations of generic agents, possibly labeled, and identical at a suitable level of description. For instance, they possess the same cognitive system, since they belong to the same species, or they use the same artificial ways of interacting. They interact in the same way, but at different times: using different values of variables, e.g., position; using different parameters, e.g., depending on density and topological position; or starting from different initial conditions. Cases (b) and (c) described above, apply as examples. In the second case, we consider populations of generic agents considered to be distinguishable, not only because they are labeled, but because they interact in different ways for reasons such as the use of structurally different rules (not just different values of variables, different parameters, and different initial conditions of the same analytical rule) but, for instance, different rules considering each value of direction, speed or altitude. Such rules are intended as if applied individually to any combination or sequence by each generic agent as introduced below.

- The number of generic agents may be fixed during the entire process or variable due to the entry or exit of generic agents for any reason.

- The way of interacting - called here the structure of interaction - may be the same, or not the same, during the entire process. In the first case - the same way of interacting during the entire process-generic agents interact using the same available library of rules. In the second case-different ways of interacting during the entire process-generic agents interact using different combinations of the same available library of rules as well as new rules established, for instance, by learning or environmental conditions.

We recall that a Multiple System is a coherent set of simultaneous or successive systems, modeled by the observer and established by the same elements interacting in different ways, i.e., having multiple roles simultaneously or at different times.

In other words Multiple Systems are systems with components belonging to more than one system. Collective Beings are particular cases of Multiple Systems where the components are autonomous agents, i.e., possessing cognitive systems allowing them to process information, as in swarms or markets [19]. Examples of coherent Multiple Systems are given by networked interacting computer 
systems performing cooperative tasks over the Internet and electricity networks where different systems play different roles. We underline that simultaneous belongings and roles are mainly due to multiple meanings of acquired states and values for different systems, e.g., control, supply, and regulatory systems, such as electronic devices and shared tasks for the software of networks.

The study of Multiple Systems also considers interchangeability between interacting agents to model emergent behavior, for instance, as being ergodic. It is well known that ergodicity is a refined recurrence property of statistical systems. In our approach, this word has a parallel and different meaning. By ergodic we mean, in this case, the possibility of identifying kinds of regularity, which define coherence in a sequence of configurations [20]. Coherence corresponds to the acquisition of an emergent property by the Multiple System. Sequences of states adopted by corresponding sequences of various single systems are established over time by the same elements interacting in different ways. For example, having variable structures establish coherent Multiple Systems acquiring, for instance, the emergent property of a black-out in electricity networks or coherent Collective Beings acquiring, for instance, the emergent properties of traffic jams, over or under-selling and the congestion of phone lines. Their quasi-coherence is considered as being due to quasi-ergodic multiple and interchangeable roles, such as values of speed, altitude, distance, and topological position in a flock of boids [23].

\section{What Happens in-between?}

As introduced above, we will consider the multiplicity of rules, which can be adopted by generic agents in any combination and for various periods of time. The problem in representing such multiplicity is almost intractable at a microscopic level where each rule should be, for instance, formalized in the same way if not limited to different values of a parameter. At a macroscopic level, we lose all the microscopic information, as when one considers statistical properties. We consider the mesoscopic level of description as an intermediate between micro and macroscopic levels and where not all the microscopic information is lost nor considered in its entirety as in Synergetics.

The invention by the observer of a suitable mesoscopic level of description is related to its constructivist role, which in this way is theoretically embedded in this approach intended as a research strategy. For instance, mesoscopic variables may be identified by considering clusters of microscopic variables used for components and at suitable threshold values by assuming a kind of Gestalt continuity. Examples are given by considering the number of generic agents establishing clusters where they have the same, at suitable thresholds, distance, speed, altitude, direction and multiple belongings are possible.

On the other hand, mesoscopic clustering, allowing identification of mesoscopic variables, may be given without assuming Gestalt continuity but by considering completely new approaches. Examples are given by considering in SCBs topological distances, scale-freeness, power laws, and even macroscopic variables such as measures of their density, surface, and behavior.

Such a mesoscopic level of description is suitable to represent what happens between macroscopic events, such as changes in values adopted by behavioral variables and regimes of validities of the rules of interaction.

As stated above, the dynamics of such kinds of complex systems are known only a posteriori and the idea to zip the essential characteristics of change into a set of ideal equations, typically a 
Lagrangian or Hamiltonian formulation based on general symmetry or conservation principles, is unsuitable. As power laws and scale-freeness are clues of complexity, i.e., the occurrence of processes of emergence and self-organization, properties of the behavior of systems selecting from among equivalent possibilities, for instance, respecting the degrees of freedom, may profile complex behaviors. The idea is to consider how systems use the available degrees of freedom. The ways of using the degrees of freedom may be represented in several ways such as considering their percentages and distributions; their statistical, periodical and quasi-periodical properties of the available interval of freedom, i.e., [max-min]. It must also be remarked that in many ideal physical systems, the concepts of "coherence", "information" and "entropy" are closely linked to the nature of the system and the kind of methodology used, such as the considered scale and the type of "environment" defined for the system. They are model-based concepts. In the case of Meta-Structures, and in particular in (SCBs) Spatial Collective Behaviors, such concepts are also defined according to the choices of resolution, aim and goal of the observer (agent-based). It is clear that such an approach does not aim to predict or simulate, but to intervene and manage. That is, to identify configurations and regularities with an ability to show us how to maintain and/or modify a process.

\section{Modeling Mesoscopic Change}

The coherence of sequences of configurations in collective behavior is represented by meta-structural properties. In this view:

- Mesoscopic variables transversally intercept and represent values adopted by aggregates of microscopic variables. Values of mesoscopic variables are considered to represent the effective application of interaction rules.

- Properties of sets of such values represent the coherence of sequences of configurations, i.e., the collective behavior.

Multiple structures are considered to be suitably represented by the values and properties adopted by mesoscopic variables, thereby specifying effective applications of rules of interaction, such as, at a suitable threshold. In the case of SCBs, for example:

(1) $M m x\left(t_{i}\right)$ number of elements having the maximum distance at a given point in time;

(2) $M n\left(t_{i}\right)$ number of elements having the minimum distance at a given point in time;

(3) $M d\left(t_{i}\right)$ number of elements having the same distance from the nearest neighbor at a given point in time;

(4) $M s\left(t_{i}\right)$ number of elements having the same speed at a given point in time;

(5) $\operatorname{Mdir}\left(t_{i}\right)$ number of elements having the same direction at a given point in time;

(6) $M a\left(t_{i}\right)$ number of elements having the same altitude at a given point in time;

(7) $M t\left(t_{i}\right)$ number of elements having the same topological position at a given point in time;

(8) Macroscopic variables such as measures of $\operatorname{Vol}\left(t_{i}\right)$, volume of the collective entity over time (used to compute density) and $\operatorname{Sur}\left(t_{i}\right)$, surface of the collective entity over time.

We specify that various same values may be valid for the same variable identifying, for instance, different clusters of elements corresponding to elements respectively at the same distances $d_{1}, d_{2}, \ldots$, 
$d_{n}$. Furthermore the same elements may belong to different clusters related to the same mesoscopic variable and to different mesoscopic variables.

Specific generic agents establish Mesoscopic variables. Sets of generic agents establishing mesoscopic variables may even be considered for other properties than their mesoscopic belonging. For instance, sets of generic agents establishing single specific or even multiple mesoscopic variables may be considered for their geometrical and statistical distributions and characteristic patterns.

We may consider a number $k$ of generic agents $e_{k}$ establishing a collective system, and $m$ is the mesoscopic property considered, for instance $m_{j: 1-M}$ where $M$ is the total number of mesoscopic variables available. In this exemplified description the numbers $k$ and $\mathrm{M}$ are considered fixed during all the phenomenon in a discretized time $t_{i: 1-T}$ where $T$ is the total time.

It is possible to introduce the mesoscopic general vector

$$
M_{m, j}\left(t_{i}\right)=\left[m_{1}\left(t_{i}\right), m_{2}\left(t_{i}\right), \ldots, m_{M}\left(t_{i}\right)\right]
$$

where $m_{j}\left(t_{i}\right)$ takes the value of 0 if no generic agents possess the mesoscopic property $m_{j}\left(t_{i}\right)$ at time $t_{i}$ taking, in other cases, the number of generic agents possessing the mesoscopic property $m_{j}\left(t_{i}\right)$ at time $t_{i}$.

It is also possible to consider a matrix $K x M\left(t_{i}\right)$ given by generic agents $e_{k}$ for mesoscopic variables where the element $K M_{k, m}\left(t_{i}\right)$ is equal to 0 if the generic agent $e_{k}$ does not possess the mesoscopic property $m$ at time $t_{i}$ or 1 if the generic agent $e_{k}$ does possess the mesoscopic property $m$ at time $t_{i}$ :

$$
\left[\begin{array}{llll}
K M_{11} & K M_{12} & \ldots & K M_{1 m} \\
K M_{21} & K M_{22} & \ldots & K M_{2 m} \\
\ldots \ldots & & & \\
K M_{k 1} & K M_{k 2} & \ldots & K M_{1 m}
\end{array}\right]
$$

This matrix - mesoscopic general vector-fixes the properties valid for single generic agents over time. The values of the vector in the matrix considered above over time are termed meta-elements.

Mathematical properties of sets of meta-elements over time are termed here as meta-structural properties, i.e., mesoscopic properties during the change satisfying the modeler's criteria and thus representing the searched emergence in the form of proper SCBs.

Examples of meta-structural properties are:

(1) Properties of the values acquired by mesoscopic variables, single or crossed, such as any regularities including periodicity, quasi-periodicity, chaotic regularities possibly with attractors which characterize specific collective behaviors;

(2) Possible statistical properties of sets of meta-elements detected by suitable techniques like Principal Components (PCs), Recurrence Quantification Analysis (RQA), Multivariate Data Analysis (MDA), Cluster Analysis, Principal Component Analysis (PCA), Time-Series Analysis, Pearson Product Moment Correlation Coefficient (PPMCC);

(3) Properties, e.g., geometrical and statistical, of sets of generic agents constituting mesoscopic variables;

(4) Properties related to the usage of degrees of freedom as introduced above; 
(5) Relationships between properties of sets of clustered generic agents and, macroscopic properties such as density, distribution, scale-freeness, numerical properties such as percentages;

(6) Properties of the thresholds adopted for specifying the mesoscopic general vector;

(7) Levels of ergodicity or quasi-ergodicity;

(8) Properties of values of the vector and the matrix considered above over time;

(9) Possible topological properties of network representations such as power laws and scale-freeness.

By the Matrix (2) it is possible to consider some structural cases, which can specify collective systems and be considered as meta-structural properties given by mesoscopic dynamics. It is possible to consider the reducibility to structural cases by properly acting upon thresholds and clustering.

We may say that meta-structures are a conceptual framework for integrated and dynamical usages of different models corresponding, for instance, to different clustering's and properties.

More generally, meta-structures allow detections of "changes" which cannot be reduced to "evolutions" [24].

When focusing on generic agents we may consider various cases of different complexities:

(1) All of the generic agents simultaneously possess all the same single mesoscopic property which is constant over time;

(2) All of the generic agents simultaneously possess the same subset, constant over time, of the mesoscopic properties available;

(3) All of the generic agents simultaneously possess a subset, variable over time, of the mesoscopic properties available;

(4) A significant percentage of the generic agents simultaneously possess all the same single mesoscopic property which is constant over time;

(5) A significant percentage of the generic agents simultaneously possess the same subset, constant over time, of the mesoscopic properties available;

(6) A significant percentage of the generic agents simultaneously possess a subset, variable over time, of the mesoscopic properties available;

(7) Any combinations of the previous cases may occur regarding different or the same generic agents.

Since the seven cases above are very general, it is necessary to specify by considering in Case (2) the subsets; in Case (3) the variability of subsets; and how generic agents are clustered by a mix of Cases (4-6), that are possibly intersected and have relative percentages.

It would be interesting to study, for instance, the possible correspondence between such cases and the topological roles of generic agents, e.g., at the center, on the boundary, in the front of or at the bottom, in the case of collective motion; or diffused.

A general view is presented in Table 1 .

Table 1. Mesoscopic dynamics.

\begin{tabular}{|c|c|c|}
\hline \multicolumn{3}{|c|}{ Mesoscopic Dynamics } \\
\hline & Structural cases & Meta-structural properties \\
\hline (1) & $\begin{array}{l}\text { All of the generic agents simultaneously possess all the same } \\
\text { single mesoscopic property constant over time; }\end{array}$ & $\begin{array}{l}\text { Trivial meta-structural properties } \\
\text { Collective behaviors structurally "fixed" }\end{array}$ \\
\hline
\end{tabular}


Table 1. Cont.

\begin{tabular}{|c|c|c|}
\hline \multicolumn{3}{|c|}{ Mesoscopic Dynamics } \\
\hline & Structural cases & Meta-structural properties \\
\hline (2) & $\begin{array}{l}\text { All of the generic agents simultaneously possess the same } \\
\text { subset, constant over time, of the mesoscopic properties } \\
\text { available; }\end{array}$ & $\begin{array}{l}\text { Trivial meta-structural properties } \\
\text { Collective behaviors structurally at } \\
\text { low variability }\end{array}$ \\
\hline (3) & $\begin{array}{l}\text { All of the generic agents simultaneously possess a subset, } \\
\text { variable over time, of the mesoscopic properties available; }\end{array}$ & $\begin{array}{l}\text { Significant meta-structural properties } \\
\text { Collective behaviors structurally variable }\end{array}$ \\
\hline (4) & $\begin{array}{l}\text { A significant percentage of the generic agents simultaneously } \\
\text { all possess the same single } \\
\text { mesoscopic property constant over time; }\end{array}$ & $\begin{array}{l}\text { Non-trivial meta-structural properties } \\
\text { Collective behaviors mesoscopically fixed }\end{array}$ \\
\hline (5) & $\begin{array}{l}\text { A significant percentage of the generic agents simultaneously } \\
\text { possess the same subset, constant over time, of the mesoscopic } \\
\text { properties available; }\end{array}$ & $\begin{array}{l}\text { Non-trivial meta-structural properties } \\
\text { Collective behaviors mesoscopically variable }\end{array}$ \\
\hline (6) & $\begin{array}{l}\text { A significant percentage of the generic agents simultaneously } \\
\text { possess a subset, variable over time, of the mesoscopic } \\
\text { properties available; }\end{array}$ & $\begin{array}{l}\text { Non-trivial meta-structural properties } \\
\text { Collective behaviors mesoscopically at } \\
\text { high variability }\end{array}$ \\
\hline (7) & $\begin{array}{l}\text { Any combinations of the previous cases may occur regarding } \\
\text { different or the same generic agents. }\end{array}$ & $\begin{array}{l}\text { Complex multiple meta-structural properties } \\
\text { Topological correspondences }\end{array}$ \\
\hline
\end{tabular}

Table 2 provides a summary of some key concepts used.

Table 2. Summary of key concepts used in the meta-structures approach.

\begin{tabular}{|l|l|}
\hline Mesoscopic variables & $\begin{array}{l}\text { Mesoscopic state variables are invented by the observer in a constructivist } \\
\text { manner and represent clusters of agents taking on the same values at the same } \\
\text { time. For instance, the value taken by a mesoscopic state variable at time } t_{i} \\
\text { represents the number of elements which have the same (within a range of } \\
\text { values) value of some microscopic state variables such as the same distance } \\
\text { from their nearest neighbors, the same speed, the same direction or the same } \\
\text { altitude over time. }\end{array}$ \\
\hline Meta-elements & $\begin{array}{l}\text { Meta-elements are time-ordered sets of values in a discrete temporal } \\
\text { representation adopted by mesoscopic variables over time and specifying } \\
\text { mesoscopic state variables, e.g., values of the same distance, speed or altitude } \\
\text { adopted by elements having this mesoscopic property. }\end{array}$ \\
\hline Meta-structural properties & $\begin{array}{l}\text { Meta-Structural properties are given by the mathematical properties possessed } \\
\text { by ordered sets of values establishing Meta-elements, e.g., statistical, periodic, } \\
\text { or correlative. }\end{array}$ \\
\hline Meta-Structure & $\begin{array}{l}\text { The term Meta-Structure relates to simultaneous multiple structures governing } \\
\text { interactions between generic agents and their sequences establishing } \\
\text { corresponding coherent sequences of spatial configurations. }\end{array}$ \\
\hline Dynamical Systems & $\begin{array}{l}\text { Fixed } \text { analytical representations of the rules of interaction between microscopic } \\
\text { or macroscopic state variables used to model a dynamic system are assumed to } \\
\text { be the structures of the system, e.g., the Brusselator, Lorenz or } \\
\text { Lotka-Volterra equations. }\end{array}$ \\
\hline
\end{tabular}




\section{Acting on Collective Behaviors}

We can consider meta-elements and meta-structural properties useful for characterizing collective behaviors as represented by their mesoscopic changing.

The metastructural approach aims to identify some configurations among all those available and equivalent within the nature of the system. The approach must induce proper selections among all equivalent configurations without forcing any of them, according to observer criteria.

As we said, the main aim of MS approach is not predicting but being an intervention on systems:

- Induce coherence within sets of elements interacting collectively giving rise to processes of emergence;

- Change properties of collective behaviors, allow merging processes;

- Maintain or restore the coherence of a collective behavior when possible changes or a loss of coherence occur for any internal and/or external reason;

- Destroy or prevent the coherence and related processes of emergence which should be avoided under any circumstances.

An example consists in inserting a perturbative collective behavior inside the collective behavior to be modified. In this context, we recall the processes of delocalization and restructuring in damaged brains. This conceptually corresponds to set fixed or variable obstacles, e.g., for collective motion asking the system for a collective, coherence-preserving reaction. Aspects of the phenomenon may be simulated by using Neural Networks. Examples of creative usages of such approaches are available [25].

For the introduction of the Perturbative Collective Behavior (PCB), we may consider aspects such as:

- Component generic agents of the PCB, may be the same generic agents of the collective behavior, mutated by another collective behavior, assuming at a certain instant, i.e., by using different interaction rules. Other component elements of the original collective behavior are invisible to the component elements of the PCB. The other component elements of the original collective behavior will interact following the constraints corresponding to the new muted agents. The PCB strategy can be applied in many different ways:

(1) The distribution of PCB agents may be fixed or variable with some regularities;

(2) The percentages of agents acquiring mutations may be fixed or variable and have a fixed duration or be properly distributed over time;

(3) It is possible to obtain various simultaneous or subsequent PCBs.

- The possibility of considering feedback between PCB and SCB, allowing the former to change in number, properties and allowing some kind of intelligent learning regulating, according to the expected modification, to be induced.

A typical methodology, for example, consists in inserting-according to a proper space-time grid - the muted agents in CB following a doping strategy inspired by doping materials like silicon. In Condensed Matter Physics it is well known for the introduction of environmental dynamics, deformations and topological defects such as walls, dislocations, or vortices [26]. 
The nature of the possible general approach discussed above recalls effects, which can be considered in populations of cells, when some of them mutate, acquiring different properties, external agents are inserted or due to any reasons having pathological or curative pharmacological effects. Analogously, in markets or social and political groups, agents are infiltered so as to change the collective choices.

The use of such approaches to induce the emergent system to behave by respecting some specific meta-structural properties, as well as the mesoscopic dynamics of some structural cases of interest, is an important research issue.

Conceptually, we can consider at any instant the distance of values of meta-structural properties adopted by the actual collective behavior, and its recent behavioral history, starting from the hypothetically closest ones of interest for the researcher since they possess the desired meta-structural properties. We stress that different configurations may satisfy, at different mesoscopic thresholds, meta-structural requirements, and thus are equivalent from MS point of view.

The next step should be to use suitable perturbations as a kind of mesoscopic and meta-structural regulation. However, even though such regulation can be performed by following automatic procedures they should be like a learning process as well as context-sensitive, based on a variety of evolving strategies. In this context, various kinds of available, unconventional computing approaches, such as Morphing Computing, can be useful [27,28].

The above mentioned approaches constitute the research framework where we may consider the implementations of tools suitable for recognizing emergence, even in the absence of the recognition of acquired properties, and to induce, restore, reproduce or even avoid coherence.

\section{Ideal, But Not Always Practical}

The theory of Phase Transitions (PT) using the Spontaneous Symmetry Breaking (SSB) mechanism in Quantum Field Theory (QFT) is well known in the literature. Consider, for example, a marble standing in an unstable equilibrium at the top of a "Mexican Hat" potential well. When a slight variation occurs, thus breaking the equilibrium, the marble will roll down the gradient to some position in the circular valley at the bottom.

The global structure of the dynamic situation obeys general symmetry principles (the Mexican Hat does not change its shape), but the final state is highly asymmetrical. As a classical case, it is a banal situation, but if the marble considered is an infinite state quantum system and the Mexican Hat is the potential, defining its dynamic-evolutionary possibilities with infinite degrees of freedom, the question becomes quite interesting. The rolling down is really a pertinent image for radical emergence phenomena in QFT. When one of the parameters is linked to the available energy changes, the system will distribute itself in one of the many possible ground states, with a consequent energy redistribution characterizing its macroscopic properties. Each "marble position" expresses a different energy arrangement of the system, and in contrast with the classical case, there is no possibility of forecasting any details of the final state; because the renowned quantum dice, about which Einstein expressed concern, are not informationally closed with respect to the observer, whereas the statistics of quantum objects-Fermi-Dirac for fermions and Bose-Einstein for bosons-are radically different from classical statistics and provide a rich phenomenology of organized states. 
To be more precise, the key idea is that with an infinite number of states, quantum systems are different and non-unitarily equivalent representations of the same system that are possible, and consequently, phase transitions can structurally modify the system as well. This occurs by means of the $\mathrm{SSB}$, that is, a process that does not allow all the states to be compatible with a given invariant energy value [29].

What usually happens is, when a given parameter varies, the system will settle into one of its possible fundamental states, thus breaking the symmetry. This leads to balancing by the emergence of long-range correlations associated with Higgs-Goldstone bosons, which act to make the new configuration stable.

The boson-condensed states can be fully considered as forms of macroscopic coherence of the system, and they are peculiar to the quantum statistics, which formally depend upon the indistinguishability of states with respect to the observer. The new system's phase requires a new description level for its behaviors, so that we can speak of radical emergence.

Many behaviors of great interest in Physics on different scales are included within SSB processes, such as phonons in a crystal, Cooper pairs in superconductivity phenomena, the Higgs mechanism, multiple vacuum states in elementary particle physics, and inflation and formation of the "cosmic landscape" in Quantum Cosmology. It is reasonable to suppose that the fundamental processes for the formation of structures depend, essentially and critically, upon SSB and the QFT "syntax" makes it possible to grasp them.

A question of great interest arises when comparing the "ideal model" of emergence, proposed by the language of dense quantum systems, with the more classical, traditional, and "semi-classical" ones of Prigogine dissipative systems and the self-organization processes at the boundary between order and disorder. This problem is strongly correlated to the emergence of the classical world from the quantum one, and a promising approach is to consider the traditional-classical or semi-classical and critically depending on opportune boundary conditions - self-organization theories as emergent residual "traces" of SSB processes. Most of the complex systems we deal with have finite dimensions and a very high, but not infinite, number of degrees of freedom. One answer could be that these systems are the outcome of a "freezing" of the degrees of freedom typical of the SSB system and all the classical self-organization phenomena are the consequence of quantum processes of symmetry breaking. According to this idea, phenomenological emergence manifestations are a particular case of quantum radical emergence [30,31].

How can SSB radical emergence be compared with the phenomenological detection of patterns and how can the radically quantum features be distinguished? In SSB processes, the phase transition is likewise led toward a globally predictable state by an order parameter, that is, we know that there exists a critical value beyond which the system will find a new state and exhibit macroscopic correlations, and here too, a relevant role is played by boundary conditions (all in all, a phonon is the dynamic emergence occurring within a crystal lattice and it does not make any sense out of it). Moreover, in SSB, there exists an "adjustment" transient phase whose description is mostly classical. Where the analogy fails and we can actually speak of an irreducibly nonclassical feature, is the bosonic condensation, which is a non-local phenomenon. In a classical dissipative system, we can, in principle, obtain information on the "fine details" of bifurcation and know where the marble will fall, whereas in SSB processes, this is not possible because of the very nature of the quantum roulette! This point 
suggests that QFT is the conceptually "ideal" framework to describe emergence, but much less practical in many classical cases, quite common in the "middle way" [11].

In Haken's Synergetics [12] the behavior of a system close to a critical point has been hypothetically considered as being analogous to that of a system undergoing a phase transition. This hypothesis, known as the adiabatic approximation, implies that the amplitudes of fluctuations in all stable modes can be expressed in terms of the amplitude of fluctuation in only an unstable mode. Haken's approach revealed itself to be precious in the study of many meta-stable systems with relatively "quiet" phase transitions. Overall, both in Synergetics and QFT the approach to phase transitions, the asymptotic state of a system is always possible; and although nothing detailed can be said about transient dynamics, it is possible, by means of suitable order parameters, to evaluate, in quite a detailed way, the step from one phase to another. In our approach, we take into consideration cases where such evaluations are not possible even in principle. It is much more convenient to choose methods which can reveal a posteriori coherence and emergence as the characterization of the new state. In our approach, mesoscopic variables are not due to processes of adiabatic approximation, but are invented by the observer within the framework of its constructivist role $[32,33]$.

Finally, we observe that generally, in classical and quantum phase transitions, the stochastic and constructive role of fluctuations is well understood, and "domesticated" by a rich tradition of mathematical methods. As we have already mentioned, the mesoscopic scale is defined as the area of "negotiation" between micro and macro dynamics, and this area is fully defined by the global boundary conditions and microscopic interactions. These aspects allow their treatment, using ideal models. In the case of the collective systems studied by meta-structures, such as groups, swarms and flocks, the mesoscopic, and therefore, fluctuations, have an irreducible centrality still not fully understood. For example, the changes in an observable can have influence on certain aspects of the system and not on others. In particular, this depends on the fact that the inter-relations between agents do not follow simple laws of interaction, and often the effectiveness of a fluctuation depends critically on the existence of an agent-hub. However, the confrontation between methods of soft-matter, macro domains of quantum coherence and network theory remains, therefore, a problematic and open issue [34-38].

\section{Conclusions}

The fundamental lesson of complex systems is that they cannot be zipped in a single formal model. Emergence processes require the dynamic and integrated use of many different models. In this work, we introduced the general lines of an approach useful when in handling radical emergence, when we cannot make use of the traditional models of Theoretical Physics. They are collective behaviors - unpredictable on the basis of microscopic information (even when it is available) - and systems, which can be neither modeled by a "state equation" with few macroscopic variables. Actually, we cannot speak exactly of "dynamics", but of "change" in a more general sense. Typical cases are: traffic, animal collective motion, social systems and markets. Even in these intractable cases we do not renounce to the possibility of a scientific investigation. It can be interesting, for example, to investigate the manifestation of specific and significant configurations, i.e., coherent according to a cognitive-constructivist criterion. Meta-structures just respond to the exigency to explore such kinds 
of complex systems, by using sets of mesoscopic variables, chosen according to the behavior the modeler wants to point out or on the basis of his conjectures.

The MS are useful to design collective behaviors by inducing and acting upon the emergence of collective behavior, by soft approaches, making systems use meta-structural properties as order parameters and by acting on environmental conditions, information/energy availability, and local interventions on mesoscopic dynamics. In this sense, the meta-structures should be considered as a theory of change, and its management, in collective systems. In this paper, just for simplicity reasons, we refer to SCBs, and so to spatial variables, in a discretized time. The MS approach can be extended to far more abstract and complex "phase spaces", of course. These approaches are more and more diffuse in different fields such as system biology or artificial vision $[39,40]$. For the last case it is evident that the "coherence" concept depends on how the visual tracking model has been built: "The lack of a complete and general-purpose architecture for model-based tracking can be attributed in part to the apparent problem complexity: An extreme variety of scenarios with interacting objects, as well as many heterogeneous visual modalities that can be defined, processed, and combined in virtually infinite ways, may discourage any attempt to define a unifying framework. Nevertheless, a more careful analysis shows that many common properties can be identified through the variety and properly included in a common description vocabulary for most state-of-the-art systems" (quote from [40]). The exponential increasing of the computational power in itself, is not a warranty for solving problems. Nevertheless, the problem based approaches like MS combined with computational resources can reveal hidden and subtle regularities in more complex systems.

\section{Conflicts of Interest}

The authors declare no conflict of interest.

\section{References}

1. Attanasi, A.; Cavagna, A.; del Castello, L.; Giardina, I.; Grigera, T.S.; Jelić, A.; Melillo, S.; Parisi, L.; Pohl, O.; Shen, E.; et al. Superfluid Transport of Information in Turning Flocks of Starlings. Available online: http://arxiv.org/abs/1303.7097 (accessed on 22 September 2013).

2. Licata, I. Almost-anywhere theories: Reductionism and universality of emergence. Complexity 2010, 15, 11-19.

3. Pessa, E. Physical and Biological Emergence: Are They Different? In Systemics of Emergence. Research and Development; Minati, G., Pessa, E., Abram, M., Eds.; Springer: Berlin, Germany, 2006; pp. 355-374.

4. Pessa, E. Phase Transitions in Biological Matter. In Physics of Emergence and Organization; Licata, I., Sakaji, A., Eds.; World Scientific: Singapore, 2008; pp. 165-228.

5. Giuliani, A.; Zbilut, J. The Latent Order of Complexity; NovaScience: New York, NY, USA, 2008.

6. Krause, J. Ruxton GD (2002) Living in Groups; Oxford University Press: New York, NY, USA, 2002.

7. Newman, M. Networks: An Introduction; Oxford University Press: New York, NY, USA, 2010.

8. Kadushin, C. Understanding Social Networks: Theories, Concepts, and Findings; Oxford University Press: New York, NY, USA, 2011. 
9. Viswanathan, G.M.; da Luz, M.G.E.; Raposo, E.P.; Stanley, H.E. The Physics of Foraging: An Introduction to Random Searches and Biological Encounters; Cambridge University Press: Cambridge, UK, 2011.

10. Vicsek, T.; Zafeiris, A. Collective Motion. Available online: http://arxiv.org/abs/1010.5017 (accessed on 22 September 2013).

11. Laughlin, R.B.; Pines, D.; Schmalian, J.; Stojkovic, B.P.; Wolynes, P. The middle way. Proc. Natl. Acad. Sci. USA 2000, 97, 32-37.

12. Haken, H. Advanced Synergetics: Instability Hierarchies of Self-Organizing Systems and Devices; Springer: New York, NY, USA, 1983.

13. Minati, G.; Licata, I.; Pessa, E. Meta-Structures: The Search of Coherence in Collective Behaviours without Physics. Available online: http://arxiv.org/abs/1305.0206 (accessed on 22 September 2013).

14. Minati, G. New Approaches for Modelling Emergence of Collective Phenomena; Polimetrica: Milan, France, 2008.

15. Minati, G.; Licata, I. Meta-structural properties in collective behaviors. Int. J. Gen. Syst. 2012, 41, 289-311.

16. Cruchtfield, J.P. The calculi of emergence: Computation, dynamics and induction. Phys. D 1994, $75,11-54$.

17. Licata, I.; Minati, G. Creativity as Cognitive Design: The Case of Mesoscopic Variables in Meta-Structures. In Creativity: Fostering, Measuring and Contexts; Corrigan, A., Ed.; NovaScience: New York, NY, USA, 2010; pp. 95-108.

18. Goldstein, J. Emergence as a construct: History and issues. Emergence 1999, 1, 49-72.

19. Ronald, E.M.A.; Sipper, M.; Capcarrere, M.S. Design, observation, surprise! A test for emergence. Artif. Life 1999, 5, 225-239

20. Ryan, A.J. Emergence is coupled to scope, not level. Complexity 2006, 67, 67-77.

21. Minati, G.; Pessa, E. Collective Beings; Springer: New York, NY, USA, 2006.

22. Minati, G. Emergence and Ergodicity: A Line of Research. In Emergence in Complex, Cognitive, Social and Biological Systems; Minati, G., Pessa, E., Eds.; Kluwer: New York, NY, USA, 2002; pp. 85-102.

23. Ballerini, M.; Cabibbo, N.; Candelier, R.; Cavagna, A.; Cisbani, E.; Giardina, I.; Lecomte, V.; Orlandi, A.; Parisi, G.; Procaccini, A.; et al. Interaction ruling animal collectivebehavior depends on topological rather than metric distance: Evidence from a field study. Proc. Natl. Acad. Sci. USA 2007, 105, 1232-1237.

24. Pessa, E. Towards a General Theory of Change. In Processes of Emergence of Systems and Systemic Properties. Toward a General Theory of Emergence; Minati, G., Abram, M., Pessa, E., Eds.; World Scientific: Singapore, 2009; pp. 603-623.

25. Thaler, S.L. The creativity machine paradigm: Withstanding the argument from consciousness, the American philosophical association. Newslett. Philos. Computers 2012, 11, 19-30.

26. Pessa, E. On Models of Emergent Metastructures. In Methods, Models, Simulations and Approaches towards a General Theory of Change; Minati, G., Abram, M., Pessa, E., Eds.; World Scientific: Singapore, 2012; pp. 113-134. 
27. Resconi, G.; Nikravesh, M. Morphic Computing. In Theoretical Advances and Applications of Fuzzy Logic and Soft Computing Advances in Soft Computing; Springer: New York, NY, USA, 2007; Volume 42, pp. 180-189.

28. Resconi, I.; Licata, I. Information as Environment Changings-Classical and Quantum Morphic Computation. In Methods, Models, Simulations and Approaches towards a General Theory of Change; Minati, G., Abram, M., Pessa, E., Eds.; World Scientific: Singapore, 2012; pp. 47-81.

29. Blasone, M.; Jizba, P.; Vitiello, G. Quantum Field Theory and Its Macroscopic Manifestations; Imperial College Press: London, UK, 2011.

30. Licata, I. Emergence and Computation at the Edge of Classical and Quantum Systems. In Physics of Emergence and Organization; Licata, I., Sakaji A., Eds.; World Scientific: Singapore, 2008; pp. 1-25.

31. Licata, I. Living with Radical Uncertainty. The Exemplary Case of Folding Protein. In Crossing in Complexity: Interdisciplinary Application of Physics in Biological and Social Systems; Licata, I., Sakaji, A., Eds.; NovaScience: New York, NY, USA, 2010; pp. 1-9.

32. Licata, I. Logical openness in cognitive models. Epistemologia 2008, 31, 177-192.

33. Licata, I. Seeing by Models; Vision as Adaptive Epistemology. In Methods, Models, Simulations and Approaches towards a General Theory of Change; Minati, G., Abram, M., Pessa, E., Eds.; World Scientific: Singapore, 2012; pp. 385-400.

34. Hirst, L.S. Fundamentals of Soft Matter Science; CRC Press: Boca Raton, FL, USA, 2012.

35. Bunde, A.; Havlin, S. Fractals and Disordered Systems; Springer: New York, NY, USA, 2012.

36. Newman, M.; Barabasi, A.-L.; Watts, D.J. The Structure and Dynamics of Networks; Princeton University Press: Princeton, NJ, USA, 2006.

37. Wolfram, S. A New Kind of Science; Wolfram Media Inc.: Champaign, IL, USA, 2002.

38. Schroeder, M. Fractals, Chaos, Power Laws: Minutes from an Infinite Paradise; Dover Publications Inc.: New York, NY, USA, 2009.

39. Bizzarri, M.; Palombo, A.; Cucina, A. Theoretical aspects of systems biology. Prog. Biophys. Mol. Biol. 2013, 112, 33-43.

40. Panin, G. Model-Based Visual Tracking. The OpenTL Framework; John Wiley \& Sons: Hoboken, NJ, USA, 2011.

(C) 2013 by the authors; licensee MDPI, Basel, Switzerland. This article is an open access article distributed under the terms and conditions of the Creative Commons Attribution license (http://creativecommons.org/licenses/by/3.0/). 